Revista de Negócios_ISSN 1980.4431_vol. 18, n.1, p.10_24, 2013_DOI:10.7867/1980-

431.2013v18n1p10_24

Edição Especial: Produção de Conhecimento Científico em Marketing

\title{
Perspectivas e limites da pesquisa qualitativa na produção de conhecimento em Marketing
}

\section{Prospects and limitations of qualitative research in the knowledge production in Marketing}

Francisco Giovanni David Vieira

UEM - Universidade Estadual de Maringá

GIPEM - Grupo Interdisciplinar de Pesquisas e Estudos em Marketing

PPA - Programa de Pós-Graduação em Administração

e-mail: fgdvieira@yahoo.com

Recebido em 15 de fevereiro de 2013. Aprovado em 25 de fevereiro de 2013

Editor Responsável: Edson Roberto Scharf, Dr.

Autor convidado para edição especial

\section{Resumo}

Este artigo se insere no esforço de debate sobre a produção de conhecimento em marketing no Brasil. Trata, especificamente, de perspectivas e limites do uso de pesquisa qualitativa em marketing. Apresenta um rápido panorama da publicação recente sobre produção de conhecimento em marketing no Brasil, para em seguida indicar antecedentes ao debate sobre o uso de pesquisa qualitativa na produção de conhecimento em marketing. Posteriormente pontua perspectivas e limites relacionados à pesquisa qualitativa que tanto podem ser considerados de um modo geral, quanto particularizados à produção de conhecimento em marketing. Ao final, considera-se que é preciso entender que a questão do uso da pesquisa qualitativa para a produção de conhecimento em marketing não é uma questão isolada. Ela faz parte de um conjunto de outras questões relativas a marketing enquanto área de conhecimento, para as quais ainda não se encontrou respostas definitivas ou que continuam em permanente tensão acadêmico-científica.

Palavras-chave: pesquisa qualitativa, marketing, conhecimento em marketing. 


\begin{abstract}
This article intends to be part of the debate about the production of knowledge in marketing in Brazil. Specifically, it approaches perspectives and limits of the use of qualitative research in marketing. As starting point provides a quick overview of recent publication on the production of knowledge in marketing in Brazil, and then indicates antecedents of the debate on the use of qualitative research in the production of knowledge in marketing. Following, it stands out prospects and limitations related to both qualitative research, which can be considered in general or particularly in the production of knowledge in marketing. At the end, the article argues that one should understand that the issue of the use of qualitative research for the production of knowledge in marketing is not an isolated issue. It is one of a number of other issues relating to marketing as a field of knowledge, which has not found definitive answers yet or that remain in permanent academic-scientific tension.
\end{abstract}

Keywords: qualitative research, marketing, knowledge in marketing.

"Mandei plantar Folhas de sonho no jardim do solar As folhas sabem procurar pelo sol $E$ as raizes procurar, procurar Mas as pessoas na sala de jantar (...) São ocupadas em nascer e morrer"

(Panis et Circenses, Caetano Veloso e Gilberto Gil)

\section{Introdução}

Este artigo se insere no esforço de debate sobre a produção de conhecimento em marketing no Brasil e trata especificamente, como o título sugere, de perspectivas e limites do uso da pesquisa qualitativa em marketing. Embora tenha-se testemunhado nos últimos anos a publicação de vários trabalhos que procuram compreender ou explicar a produção acadêmica em marketing em nosso país (Vieira, 1998; Vieira, 2000b; Vieira, 2003; Farias, 2004; Kovacs et. al., 2004; Rossi e Farias, 2006; Sampaio e Perin, 2006;
Sampaio et. al., 2012; Vieira, 2012; Mazzon e Hernandez, 2013), a condição do uso e da aceitação de pesquisa qualitativa por acadêmicos brasileiros permanece envolta a incompreensões e resistências, por um lado, e inconsistências na sua realização e apresentação em relatórios de pesquisa e artigos de caráter acadêmicocientífico, por outro (Sampaio et al., 2012).

Tal situação desperta a atenção e reivindica reflexão e debate porque ela transcende a questão pura e simples de eventuais problemas relacionados à realização de pesquisa qualitativa para produção de conhecimento em marketing no Brasil. Como apontado anteriormente por Froemming et al. (2000a), Froemming et al. (2000b), Sampaio e Perin (2006) e Sampaio et al. (2012), a produção científica em marketing no Brasil apresentou, e ainda tem apresentado, de um modo geral, diversas questões limitantes em sua realização. Ademais, a insistência em se destacar de forma determinante problemas relacionados à pesquisa qualitativa em marketing no Brasil sugere um incompreensível abandono ou não reconhecimento do esforço recentemente empreendido por diferentes pesquisadores brasileiros para dirimir questões e problemas de ordem métodológica e avançar na compreensão da pesquisa qualitativa em administração, em termos gerais, ou em marketing, de modo particular (Godoy, 1995; Bernardo e Vieira, 1999; Lima, 1999; Cerchiaro, Sauerbronn, e Ayrosa, 2004; Vieira e Tibola, 2005; Farias, 2006; Costa e Vieira, 2007; Rocha e Rocha, 2007; Sauerbronn e Faria, 2009; Souza Netto e Mello, 2009; Sauerbronn e Ayrosa, 2010; Mattos, 2011; Paiva Jr., Leão, e Mello, 2011; Sauerbronn, Cerchiaro, e Ayrosa, 2012).

Algumas das questões que invariavelmente vêm à baila acerca de pesquisa qualitativa em marketing concernem, por exemplo, à amplitude do campo de pesquisa qualitativa, à relação 
entre rigor e relevância na produção científica, e aos possíveis motivos da não aprovação de artigos científicos oriundos da realização de pesquisa qualitativa em marketing. Outras questões relacionadas às tendências de temas de estudo na produção científica em marketing por meio de pesquisa qualitativa, ao diálogo com outras áreas de conhecimento e sua aderência aos preceitos de marketing, ou se a produção nacional do conhecimento em marketing por meio de pesquisa qualitativa está em consonância com a produção global, também merecem ser ressaltadas. Há, assim, muitas variantes que podem servir como ponto de partida ou como itens norteadores para um debate em torno do uso da pesquisa qualitativa na produção de conhecimento em marketing. Em que pesem tais possibilidades, a abordagem realizada neste artigo não se filia a nenhuma delas de modo específico, entretanto procura tê-las como se fora uma espécie de mosaico de fundo.

No sentido de sua apresentação o artigo está dividido em quatro momentos: no primeiro momento são apontados alguns antecedentes relativos ao debate sobre a produção de conhecimento em marketing por meio de pesquisa qualitativa. No segundo e terceiro momentos são apontadas, respectivamente, perspectivas e limites da pesquisa qualitativa na produção de conhecimento em marketing. No quarto e último momento são realizadas considerações finais acerca do debate até então empreendido. Ressaltase que a abordagem realizada toma como ponto de partida a realidade da produção brasileira na área de marketing, porém não se exime de relacioná-la, pontual e circunstancialmente, à experiência acadêmico-científica que ocorre em tal área fora do Brasil. Por fim, é importante apontar que as observações e considerações tecidas ao longo do artigo têm, sobretudo, o caráter de observações e notas para debate, de modo que não têm a pretensão de assumir uma forma prescritiva e muito menos peremptória.

$\begin{aligned} & \text { Alguns antecedentes do debate sobre a } \\ & \text { produção de conhecimento em } \\ & \text { Marketing por meio de pesquisa } \\ & \text { qualitativa }\end{aligned}$.

Inicialmente faz-se necessário assinalar que o debate acerca do uso de pesquisa qualitativa para produção de conhecimento em marketing pode ser considerado, em boa medida, uma espécie de debate sobre a própria disciplina. Em outras palavras, um debate sobre a própria condição do conhecimento de marketing. Não obstante marketing, enquanto disciplina acadêmica (Saunders e Lee, 2005; Baker, 2007) e área de atuação gerencial (Hunt, 1976; Cornelissen, 2002; Faria, 2007), já tenha um longo caminho de sistematização acadêmica, científica e gerencial, o fato é que marketing continua a ser questionado em suas proposições e formulações teóricas (Zaltman, 1982; Hunt, 2002) e especialmente em sua relevância gerencial, como ocorrido recentemente por meio de edição comemorativa de 75 anos de seu mais antigo e prestigiado periódico científico, o Journal of Marketing (Jaworski, 2011).

Nesse sentido, se há um caráter controverso no que se refere à produção de conhecimento na disciplina, ele não repousa apenas sobre a pesquisa qualitativa em marketing, mas sim sobre a própria disciplina e sua capacidade de ser útil enquanto área de conhecimento (Armstrong, 2004). Isso expressa claramente que a própria área está na berlinda em termos do que produz academicamente (November, 2004). Tratase de uma importante questão que deveria minimamente orientar o debate em torno da produção de conhecimento em marketing sinalizando para uma ponderação acerca de eventuais indicações de supremacia de uma ou outra forma de 
produção de conhecimento, seja ela qualitativa ou quantitativa.

É preciso aquiescer que essa não é uma situação confortável ou desejada para a disciplina. Não são apenas pesquisadores que trabalham com pesquisa qualitativa que estão sob pressão e precisam demonstrar consistência nas suas preferências e no resultado das suas escolhas. É a própria disciplina que precisa se legitimar. A propósito, essa condição vai além dos limites da disciplina de marketing. A Administração em si, enquanto área acadêmica e de atuação, que é aplicada em sua concepção e proposição final, tem sistematicamente produzido um conhecimento que não tem alcançado os praticantes. E isso não ocorre só no Brasil, como também em outros países com economias mais avançadas (Kelemen e Bansal, 2002). Portanto, parece ser necessário refletir sobre a relevância da disciplina de marketing (Wensley, 2002; Varadarajan, 2003; Faria, 2007) e sobre o propósito da pesquisa em marketing, antes de se refletir sobre a utilização, e o rigor na utilização, de pesquisa qualitativa para a produção de conhecimento em marketing.

Avançando um pouco no debate, é possível que parte dessa situação experimentada em marketing esteja relacionada à circunstancial ausência de estudo e compreensão de Filosofia da Ciência (Oliva, 1980; Alves, 1985; Lara 1986) por parte de acadêmicos de marketing (Hunt, 2002). A mínima sugestão de um envolvimento de marketing com Filosofia da Ciência pode soar como um impropério (Hunt, 2001). Isso é em parte alimentado pela urgência cada vez maior da produção de conhecimento (Vieira, 2007) e do caráter funcional da área (McLaran et al., 2010). É como se não coubesse estudar Filosofia da Ciência em um curso ou escola de business. Caracteriza-se, assim, uma espécie de aporia: o acadêmico de marketing quer produzir conhecimento em marketing, dentro de Ciências Sociais Aplicadas, mas quer fazê-lo permitindo-se o direito de abdicar do estudo de Filosofia da Ciência. Eis aí uma questão séria e de entrave para a área, pois em tese pode representar a gênese da dificuldade de ordem metodológica cujo aspecto mais visível é a existência ou não de relevância do conhecimento produzido no âmbito da disciplina.

As escolas de negócios, instituições de ensino superior, agências de fomento, empresas de consultoria, indústria editorial e os próprios acadêmicos, de algum modo, são responsáveis por esse contexto (Faria, 2001). No ponto de origem, circunscrito às instituições de ensino superior, tem-se a conformação de estruturas curriculares que priorizam a urgência, por um lado, e que se pretendem capazes de proporcionar $\mathrm{o}$ empreendimento científico ao se ancorarem em disciplinas de formação vinculadas à estatística e à modelagem matemática, por outro.

$O$ afã de se fazer ciência joga o acadêmico de marketing em um labirinto em que se acredita ser literalmente científico. Mais que isso, produz a ideia de fazer algo asséptico, onde não há espaço para o debate de concepções ontológicas, gnosiológicas ou epistemológicas (Hunt, 2003). Alimenta-se com isso o mito da neutralidade científica (Japiassu, 1975), que tanto agrada editores e revisores de periódicos e que é uma das vertentes críticas e controversas que se impõem como restrição ao uso da pesquisa qualitativa na produção de conhecimento em marketing. $O$ resultado é que tal escolha, além de se caracterizar como sectária, mostra-se praticamente inócua diante dos problemas de relevância (Wensley, 2002; Armstrong, 2004; Faria, 2007) enfrentados pela disciplina e que transcendem de longe o uso da pesquisa qualitativa.

Não obstante transcenda a circunstância da realização de pesquisa 
qualitativa em marketing, a noção de relevância surge para a pesquisa qualitativa como um adicional questionamento por rigor. Assim, pesquisadores que trabalham com pesquisa qualitativa estão constantemente sob pressão (Donmoyer, 2012). De algum modo, tais pesquisadores precisam fazer esforço considerável para demonstrar a relevância dos trabalhos que realizam. As contribuições oferecidas por pesquisas qualitativas são muitas vezes consideradas insuficientes por editores e revisores. Há a ideia de que uma contribuição existe quando se apresenta uma teoria ou um modelo, o que é mais simples e frequente em estudos quantitativos (Huy, 2012).

Aparentemente velado, existe um embate que se dá nos processos editoriais e de revisão em periódicos (Vieira, 2000a; Huy, 2012), assim como há um processo de desgaste e relativa tensão em processos de seleção de candidatos a programas de pós-graduação que tenham trabalhado previamente com pesquisa qualitativa (Vieira, 2000a). Há aí, não apenas uma questão de ordem metodológica, como também a concepção do que é mainstream na e para a área, mesmo a despeito de existir no Brasil uma contínua produção de conhecimento em marketing por meio de pesquisa qualitativa (Sampaio et al. 2012).

De outro modo, é possível depreender desse embate a existência de uma disputa política em termos de concepções de como se faz ciência ou se produz conhecimento em marketing no Brasil. O espaço da produção e do debate acadêmico, assim como todo e qualquer espaço público, é um espaço de poder. As articulações em torno da ocupação desse espaço e da busca de legitimidade em tal ocupação, existem e não podem ser escamoteadas. Em outras palavras, como pano de fundo do debate sobre pesquisa qualitativa e pesquisa quantitativa está um embate entre formas de produzir conhecimento. E, como se sabe, conhe- cimento e poder se pressupõem mutuamente. Nesse sentido, ocupação do espaço público e visibilidade editorial também é uma forma de poder. O que se coloca é: o que há de tão restritivo no conhecimento em marketing produzido por meio de pesquisa qualitativa?

A questão do local, daquilo que é a referência empírica na pesquisa qualitativa, é uma restrição que se pode ter como um antecedente para o debate. Critica-se com grande assiduidade a incapacidade de generalização da pesquisa qualitativa (Alencar, 1999; Alves-Mazzotti e Gewandszajder, 1999; Yin, 2001; Donmoyer, 2012; Huy, 2012), sobretudo ao se lançar mão do argumento de que, via de regra, ela é conduzida envolvendo poucos sujeitos de pesquisa e em um espaço geográfico restrito. Relaciona-se generalização com lócus e, assim, criticase o lugar do lugar na pesquisa qualitativa em marketing. O lugar deixa de ser importante por restringir o alcance da pesquisa e não permitir a generalização.

Há aí uma concepção equivocada de que o local não é importante ou relevante. Essa concepção é forjada na proposição de que aquilo que é ou representa o local tem pouco para oferecer ao que está em outro espaço. Essa ansiedade na forma de se pensar e tentar produzir conhecimento em marketing atende claramente o interesse não só de uma perspectiva dominante, global, mas também corrobora a concepção das grandes business schools que procuram criar ou produzir uma espécie de conhecimento que supostamente possa ser aplicado em qualquer lugar. É a ideia de espalhar ao mundo, de levar a qualquer lugar. Ora, a própria Administração enquanto área, em seus primórdios, desenvolveu-se a partir de experiências muito particulares e restritas a determinados contextos (Fayol, 1994; Taylor, 1995), cujos resultados posteriormente mostraram-se passíveis de uso em 
diferentes lugares sob condições análogas. De algum modo, as realidades dos locais podem se assemelhar em diferentes aspectos ou sentidos. Ademais, a realidade brasileira, e mesmo de países do Hemisfério Norte, não é uma realidade única e exclusivamente alicerçada em grandes negócios ou corporações (Richers, 2000).

O local, portanto, é importante. De modo oposto, não é o fato de usar um procedimento estatístico, por exemplo, que garante a universalidade e a possibilidade de generalização do conhecimento produzido em marketing. Esse é um enorme equívoco que se continua a cometer. O próprio conhecimento resultante de análises de dados quantitativos é produzido, em sua base, em um local específico (Qu e Dumay, 2011). Ele não tem, de per si, o passaporte da universalidade. Paradoxalmente, em que pesem as restrições ao lugar do lugar na pesquisa qualitativa, e acadêmicos brasileiros continuem com uma agenda de pesquisa pautada em temáticas externas (Mazzon e Hernandez, 2013), é oportuno destacar que já se reconhece claramente que há a necessidade da teoria de marketing caminhar na direção de países emergentes como o Brasil (Sheth, 2011).

Espera-se que as observações realizadas até esse momento no artigo tornem mais simples e objetiva a abordagem de algumas das perspectivas e limites da pesquisa qualitativa na produção do conhecimento em marketing. É o que se procura fazer a seguir.

\section{Sobre perspectivas da pesquisa qualitativa}

Antes de qualquer coisa, é preciso compreender que a pesquisa qualitativa é um termo que não representa algo único ou monolítico, como se pode querer acreditar em um primeiro olhar. De modo contrário, o conceito de pesquisa qualitativa expressa pluralidade. Traduz um campo heterogêneo que envolve diferentes abordagens, com o uso de técnicas, procedimentos e recursos sob diferentes concepções filosóficas e metodológicas que procuram investigar, interpretar e explicar o mundo social, como, por exemplo, etnografia, etnometodologia, pesquisa ação, grounded theory e fenomenologia, entre outras (Creswell, 1994; Denzin e Lincoln, 1994; Miles e Huberman, 1994; Creswell, 1998; Bruyne, Herman, e Schoutheete, 1999; Fetterman, 2010; Staller, 2010). Tal caracterização representa, a um só tempo, consideráveis possibilidades e importantes desafios para a produção de conhecimento em marketing (Belk, 2006; Moisander e Valtonen, 2006).

Possibilidades, principalmente porque envolve pluralidade e diversidade perante uma realidade econômica e social cada vez mais pós-moderna, fragmentada, híbrida, hiper-real, com reversão entre produção e consumo, sujeitos descentrados e justaposição de opostos (Firat, 1993), e que, portanto, não parece comportar estruturas e procedimentos investigativos e analíticos absolutamente rígidos e prémoldados. Desafios, sobretudo porque requer clareza de concepção ontológica, capacidade de compreensão epistemológica, largo horizonte cronológico, profundidade teórica e consistência analítica. É possível que a contribuição da pesquisa qualitativa para a produção de conhecimento em marketing esteja alicerçada na reunião de tais possibilidades e desafios.

Um aspecto adicional que merece destaque refere-se ao diálogo e interação que a pesquisa qualitativa empreende com várias disciplinas que dão suporte a marketing e contribuem no sentido de formar o seu quadro teórico, como, por exemplo, Sociologia, Antropologia e Psicologia. De algum modo, tal interação recrudesce o caráter de interdisciplinaridade presente em marketing, algo que historicamente tem sido observado 
quando seus pesquisadores lançam mão de outras disciplinas na tentativa de construir um estatuto teórico particular para marketing (Lazer e Kelley, 1960).

Posto isso, destaca-se a seguir alguns aspectos e características da pesquisa qualitativa que podem ratificá-la como uma perspectiva para a produção de conhecimento em marketing e que podem ser tomados como elementos para debate por parte de editores, revisores e pesquisadores de marketing em geral.

A pesquisa qualitativa é uma jornada. Como tal, é uma experiência relativamente longa e de envolvimento direto com o método, procedimento, instrumentos, recursos e sujeitos do estudo. A presença do pesquisador em campo é algo quase que imperativo. Quando isso não acontece, seja no caso do uso de recursos eletrônicos, ou por meio de $e$ research, há um processo de interação com os sujeitos da pesquisa. Sob esse aspecto ela traz consideráveis contribuições para a formação do pesquisador de marketing. Essa condição, lamentavelmente, não é descrita ou informada nos relatórios de pesquisa e artigos submetidos para análise por parte dos periódicos - ou não é valorizada por editores e revisores.

Os sujeitos da pesquisa tendem a se engajar no processo de pesquisa qualitativa. Uma vez que os sujeitos, indivíduos ou organizações, decidem participar e se permitem ser entrevistados e fornecer informações, desencadeia-se uma colaboração. Nesse sentido, é como se o conhecimento fosse construído com eles e por meio deles (Clark, 2010). Isso é extremamente relevante para a área de marketing, embora tenha sido sistematicamente desconsiderado e até mesmo ignorado.

Há uma preocupação crescente com critérios de qualidade e validade na pesquisa qualitativa. Desde critérios de qualidade relativos à pesquisa qualitativa como um todo (Easterby-Smith, Golden-
Biddle, e Locke, 2008; Koro-Ljungberg, 2010; Tracy, 2010), até critérios exclusivos à realização de entrevistas. Particularmente no que se refere às entrevistas, há protocolos que, uma vez seguidos, contribuem no sentido de trazer consistência aos resultados finais da pesquisa qualitativa (McCracken, 1988; Roulston, 2010). Essa é uma observação importante porque a realização de entrevistas pode ser facilmente objeto de críticas quanto a encantamento, fascínio, pressa, manipulação, falta de objetividade ou não abordagem do problema central por parte do entrevistador (Watson, 2009; Roulston, 2010).

A pesquisa qualitativa apresenta uma condição única de captar e compreender questões que não se quer revelar. Devido ao processo de imersão e uso de triangulação de dados e informações, a pesquisa qualitativa oferece a possibilidade de se aproximar de questões sensíveis aos interesses de diferentes agentes que operam no mercado, desde o Estado até grandes companhias transnacionais (Vieira et. al., 2002; Mendes, Vieira, e Chaves, 2009).

A pesquisa qualitativa é dinâmica $\mathrm{e}$ tem se inovado constantemente nos últimos anos. Talvez por sua condição pluralista (Easterby-Smith, Golden-Biddle, e Locke, 2008), a pesquisa qualitativa apresenta diferentes métodos, recursos e narrativas que têm incorporado recursos tecnológicos e novos protocolos de pesquisa relacionados à videografia, fotografia, informática e internet. Tal incorporação tem incrementado e produzido maior qualidade na pesquisa qualitativa, como se tem observado, por exemplo, por meio da sofisticação no uso de grupos de foco, $e$ research, softwares e rastreamento de discurso e pesquisa de símbolos e imagens (O'Toole e Were, 2008; LeGreco e Tracy, 2009; Wiles, Crow e Pain, 2011; KoroLjungberg, 2012). 
Uma vez assinaladas algumas perspectivas que, espera-se, possam ser tomadas como elementos para debate acerca da contribuição da pesquisa qualitativa para a produção de conhecimento em marketing, a próxima seção, por outro lado, trata de alguns de seus limites.

\section{Sobre limites da pesquisa qualitativa}

A pesquisa qualitativa tem estado sob escrutínio e sofre resistência por parte de acadêmicos de marketing desde meados do século passado, quando passou a ser usada de forma cada vez mais crescente em marketing (Levy, 2006). A origem de tal resistência esteve, e talvez ainda esteja, vinculada ao fato da pesquisa qualitativa ser associada à Antropologia, Sociologia, História e Psicologia (Daymon e Holloway, 2002; Levy, 2006), distanciando-se da Economia, que exerceu forte influência na disciplina de marketing no século XX (Sheth, Gardner, e Garrett, 1988). É como se fosse considerada um processo estranho e inadequado de produzir conhecimento em escolas de negócios e mesmo em firmas de consultoria.

A rigor, essa é uma condição paradoxal, pois ao que interessou a disciplina de marketing, a associação inicial foi útil e frutífera, particularmente do ponto de vista teórico (Jonassen, 1959; Lazer e Kelley, 1960; Winick, 1961). Não deveria haver, portanto, um processo de distanciamento metodológico abrupto para com as referidas disciplinas. Há que se convir que a construção teórica decorra da investigação com emprego de método, recursos e técnicas de investigação. Visto desse modo é como se interdisciplinaridade fosse uma falácia em marketing ou não mais do que uma tentativa de reduzir seus limites ou emprestar-lhe legitimidade por meio de disciplinas que remontam ao século XVIII.
Em última análise, todo e qualquer procedimento metodológico, conjunto de técnicas ou recursos de investigação possuem alcances definidos. Trata-se de uma compreensão básica acerca das possibilidades e limites de se produzir conhecimento por meio dessa ou daquela escolha metodológica. Em outras palavras, e como ensinam os manuais de metodologia de pesquisa, um dado procedimento metodológico, em tese, deve ser mais ou menos adequado para responder esse ou aquele problema de pesquisa. Não obstante, como indicado em momento anterior deste artigo, especificamente na seção em que se apresentam alguns antecedentes ao debate sobre a produção de conhecimento em marketing por meio de pesquisa qualitativa, a resistência à pesquisa qualitativa, bem como a busca reiterada de revelar suas fragilidades, envolvem questões de ordem epistemológica e também possuem um claro componente político de ocupação do espaço público editorial e acadêmico em marketing no Brasil.

A despeito de tais considerações, limites da pesquisa qualitativa para a produção de conhecimento em marketing podem ser trazidos ao debate. É o que se procura fazer a seguir, apontando alguns deles.

O protocolo completo de pesquisa qualitativa é longo e requer tempo. Talvez esse seja o principal limite no que concerne ao uso de pesquisa qualitativa. $\mathrm{O}$ trabalho de planejamento e execução envolve diferentes etapas em que a presença do pesquisador e interação com os sujeitos da pesquisa é imprescindível. Dependendo do procedimento de validade empregado na pesquisa, haverá a necessidade de se retornar parte do corpo de dados obtido em campo aos próprios sujeitos da pesquisa que precisarão, por seu turno, devolvê-lo ao pesquisador, que não tem certeza que tal devolução de fato ocorrerá. A interação com os sujeitos, portanto, é longa, o 
trabalho de análise de dados é meticuloso, demanda tempo e não necessariamente está em consonância, por exemplo, com prazos de agências, editais, periódicos e instituições de ensino e pesquisa. Sob esse aspecto, algo que adicionalmente cabe destacar é que a própria urgência de se produzir conhecimento em cursos de pósgraduação torna-se um obstáculo à utilização de pesquisa qualitativa.

O trabalho coletivo em pesquisa qualitativa é reduzido. Tendo em vista situações de envolvimento com trabalho de campo, continuidade de análise, além do caráter multifacetado, em diferentes etapas, a pesquisa qualitativa impõe dificuldades para o trabalho coletivo. Desse modo, a possibilidade de transferência do trabalho de pesquisa qualitativa para terceiros é consideravelmente restrita. Pressupõe-se, em termos gerais, um trabalho mais solitário. Não obstante, já se começa a buscar alternativas para essa questão (Sanders e Cuneo, 2010) com a promoção de colaboração no uso de pesquisa qualitativa por meio de equipes de pesquisadores (Paulus, Woodside, e Ziegler, 2010).

A análise de narrativas em pesquisa qualitativa é circunscrita e complexa. Há um volume considerável de informações que possuem um caráter anônimo que são concedidas por sujeitos de pesquisa. Lidar com segurança com tais narrativas e preservar a relação, ainda que circunstancial, entre entrevistado e entrevistador, impõe limites à pesquisa qualitativa (Deutscher, 1973; Tilley e Woodthorpe, 2011). Isso é recrudescido para a construção da pesquisa qualitativa quando se trata, principalmente, da obtenção de informações confidenciais sobre questões éticas (Iphofen, 2011) de ordem individual ou institucional.

Há extensa descrição em pesquisa qualitativa. Talvez em função da já conhecida resistência à pesquisa qualitativa, pesquisadores têm se preocupado com descrições em detrimento de profundidade analítica. Com isso, correm o risco de não evidenciar claramente a construção teórica resultante de suas pesquisas.

A condição de coadjuvante da pesquisa qualitativa em métodos mistos. Ainda que o uso de métodos mistos seja relevante para a confirmação, amplitude e visibilidade da pesquisa qualitativa (Harrison e Reilly, 2011), o papel assumido pela pesquisa qualitativa na adoção de métodos mistos tem sido de coadjuvante. A questão mais importante sob esse aspecto, no entanto, diz respeito ao fato de a pesquisa qualitativa sofrer críticas ou restrições relacionadas à conexão positivista com métodos mistos (Hesse-Biber, 2010), onde é possível observar um esvaziamento do propósito da análise interpretativa.

Espera-se que os limites acima assinalados possam ser objeto de ponderação e reflexão ao debate e, sobremodo, ao exercício da crítica que se faz a respeito de pesquisa qualitativa como forma de produção de conhecimento em marketing.

\section{Considerações finais}

É preciso entender que a questão do uso da pesquisa qualitativa para a produção de conhecimento em marketing não é isolada. Ela faz parte de um conjunto de outras questões relativas a marketing enquanto área de conhecimento, para as quais ainda não se encontrou respostas definitivas ou que continuam em permanente tensão acadêmico-científica.

Lamentavelmente, o debate e, sobretudo, a busca de compreensão acerca de procedimentos de pesquisa qualitativa ainda é bastante tímido no Brasil. A resistência à pesquisa qualitativa é surpreendente e em boa medida inexplicável e injustificável. $O$ processo de busca de legitimidade, no Brasil, para o conhe- 
cimento produzido em marketing por meio de pesquisa qualitativa parece ter uma longa estrada e talvez seja apenas obtido no futuro e com novas gerações de pesquisadores. Atualmente, fora do Brasil, importantes periódicos como o Journal of Marketing Management, Journal of Business Research, Journal of Consumer Research e European Journal of Marketing, entre outros, têm publicado com regularidade artigos resultantes do uso de pesquisa qualitativa em marketing. Espera-se que isso possa significar alguma coisa para editores, revisores e estudiosos de marketing no Brasil.

Talvez o processo de internacionalização da produção acadêmica de marketing desenvolvida no Brasil passe pela pesquisa qualitativa. Por suas características, a pesquisa qualitativa tem forte vínculo e implicação com aquilo que, pelo menos em um primeiro momento, é local. A consolidação da área de marketing, no Brasil, e o reconhecimento de sua excelência acadêmica, certamente não serão construídos com base em protocolos de pesquisa ou temas que são contínua e exaustivamente mimetizados e replicados. Não há originalidade nisso. Talvez haja rigor. É pouco provável que exista relevância.

Nesse sentido, é necessária a definição de uma agenda de pesquisa que reflita o que é local e não o que é global. A produção de conhecimento e a capacidade de explicação de questões de marketing pautadas em uma realidade imediata de atuação, seja ela local, regional ou nacional, é ou deveria ser a prioridade. Sob esse aspecto, pela proximidade com os sujeitos de pesquisa, pelo envolvimento direto do pesquisador com o trabalho de campo e pela imersão na realidade a ser investigada, a pesquisa qualitativa pode ser extremamente importante. A contribuição decorre da originalidade, da diversidade ou do acréscimo ao conhecimento. Ela será mais frutífera quanto mais estiver pautada em originalidade e diversidade. É importante reiterar que a pesquisa qualitativa tem condições de oferecer importantes contribuições para alcançar esse propósito.

Em última análise, o rigor e, sobretudo, a relevância não são exclusividades de uma ou outra forma de pesquisa. A pesquisa qualitativa oferece condições para a investigação científica sob critérios de rigor metodológico. A definição da relevância a ser obtida com a sua utilização é uma questão de avaliação do estágio teórico alcançado sobre determinado tema de investigação; da urgência e da demanda apresentada por sujeitos de pesquisa - sejam eles indivíduos ou organizações; da complexidade e soluções encontradas em termos de referências empíricas; da oportunidade ou daquilo que se entrega como resultado passível de aplicação por praticantes, entre outras tantas diferentes possibilidades. A propósito, temáticas, tais como, cultura e consumo, gênero, identidade, resistência, marketing e sociedade, marketing social corporativo, comunidades de consumo, marketing e empreendedorismo, consumo jovem e infantil, história de marketing, consultoria em marketing e responsabilidade social corporativa, entre outras, parecem temáticas férteis para o uso de pesquisa qualitativa.

A noção geral que se tem de pesquisa qualitativa é que ela se resume à realização e análise de entrevista. Isso, de fato, por si só, não transmite credibilidade, sobretudo em um momento em que há uma grande pressão institucional para que se pesquise muito e se publique mais ainda. A pesquisa qualitativa exige maturação, reivindica ir e vir e um tempo maior de maturação do que outros tipos de pesquisas. Evidentemente, isso requer uma atenção diferenciada quanto ao seu uso.

É preciso avançar no ensino de pesquisa qualitativa em marketing. Há 
mais técnicas e recursos disponíveis hoje do que havia no passado. Isso gerará um ciclo virtuoso. Há redes de pesquisadores na world wide web que trabalham exclusivamente com métodos de pesquisa. São verdadeiros fóruns para a interação, troca de ideias e compartilhamento de soluções. Tais recursos podem favorecer, em particular, o resultado final das pesquisas. $\mathrm{O}$ ensino melhorará a produção de conhecimento em marketing por meio de pesquisa qualitativa, que certamente refletirá em uma melhoria no processo de ensino. $\mathrm{O}$ avanço no uso da pesquisa qualitativa em marketing não só é possível, como é factível.

\section{Referências}

ALENCAR, E. Introdução à metodologia de pesquisa social. Lavras: UFLA, 1999.

ALVES, R. Filosofia da ciência: introdução ao jogo e suas regras. São Paulo: Brasiliense, 1985.

ALVES-MAZZOTTI, A. J.; GEWANDSZAJDER, F. O método nas ciências naturais e sociais: pesquisa quantitativa e qualitativa. São Paulo: Pioneira, 1999.

ARMSTRONG, J. S. (2004). Does an academic research paper contain useful knowledge? In Australasian Marketing Journal, v. 12, n. 2, p.62-63, 2004.

BAKER, M. J. Business and mangement research. Helensburgh: Westburn Publisher, 2007.

BELK, R. W. (Ed.) Handbook of qualitative research methods in marketing. Northampton: Edwar Elgar, 2006.

BERNARDO, E. E. R.; VIEIRA, F. G. D. Pesquisa em administração: uma introdução ao processo e às técnicas qualitativas. Anais - Semana do Administrador, Maringá, PR, Brasil, 1999.
BRUYNE, P. de, HERMAN, J.; SCHOUTHEETE, M. de. Dinâmica da pesquisa em ciências sociais: os pólos da prática metodológica. Rio de Janeiro: Francisco Alves, 1999.

CERCHIARO, I., SAUERBRONN, J.; AYROSA, E. Uma visão alternativa da pesquisa em marketing: como a fenomenologia pode contribuir para gerar conhecimento de marketing. Anais do Encontro de Marketing da ANPAD, Porto Alegre, RS, Brasil, 2004.

CLARK, T. On 'being researched': why do people engage with qualitative research? Qualitative Research, v.10, n.4, p.399419, 2010.

CORNELISSEN, J. Academic and practioners theories of marketing. Marketing Theory, v.2, n.1, p.133-143, 2002.

COSTA, C. R. F. da; VIEIRA, F. G. D. Marketing no Brasil: pensamento e ação sob uma perspectiva historiográfica. Caderno de Administração (UEM), v.15, n.2, p.39-48, 2007.

CRESWELL, J. W. Research design: qualitative and quantitative approaches. Thousand Oaks: Sage, 1994.

CRESWELL, J. W. Qualitative inquiry and research design: choosing among five traditions. Thousand Oaks: Sage, 1998.

DAYMON, C.; HOLLOWAY, I. Qualitative research methods in public relations and marketing communications. London: Routledge, 2002.

DENZIN, N. K.; LINCOLN, Y. S. (Eds.) Handbook of qualitative research. Thousand Oaks: Sage, 1994.

DEUTSCHER, I. What we say - what we do: sentiments e acts. Glenview: Scott Foresman Company, 1973.

DONMOYER, R. Two (very) different worlds: the cultures of policymaking and 
qualitative research. Qualitative Inquiry, v.18, n.9, p.798-807, 2012.

EASTERBY-SMITH, M., GOLDENBIDDLE, K.; LOCKE, K. Working with pluralism determining quality in qualitative research. Organizational Research Methods, v.11, n.3, p.419-429, 2008.

FARIA, A. Theorizing on networks from the standpoint of managers: revealing challenges to global management education and opportunities to local marketing academics. Proceedings of BALAS 2001 Conference - The Business Association for Latin American Studies. San Diego, CA, United States, 2001.

FARIA, A. Relevância ou rigor? GV Executivo, v.6, p.39-43, 2007.

FARIAS, S. A. (2004, novembro). Em busca da inovação no marketing brasileiro: discutindo o processo de publicação de artigos em revistas e congressos. Anais do Encontro de Marketing da ANPAD, Porto Alegre, RS, Brasil, 1.

FARIAS, S. A. Pesquisa flexível em marketing: o lado humanista de uma ciência socialmente construída e fixamente investigada. Anais do Encontro de Marketing da ANPAD, Rio de Janeiro, RJ, Brasil, 2006.

FAYOL, H. Administração industrial e geral. São Paulo: Atlas, 1994.

FETTERMAN, D. M. Ethnography: stepby-step. Thousand Oaks: Sage, 2010.

FIRAT, F. Marketing in postmodern world. European Journal of Marketing, v.29, n1, p.40-56, 1993.

FROEMMING, L. M. S.; LUCE, F. B.; PERIN, M.G.; SAMPAIO, C. H.; BEBER, S. J. N.; TREZ, G. Inventário de artigos científicos na área de marketing. Revista de Administração Contemporânea, v.4, n.2, p.159-173, 2000a.

FROEMMING, L. M. S.; LUCE, F. B.; PERIN, M.G.; SAMPAIO, C. H.; BEBER,
S. J. N.; TREZ, G. Análise da qualidade dos artigos científicos da área de marketing no Brasil: as pesquisas survey na década de 90. Revista de Administração Contemporânea, v.4, n.3, p.201-219, 2000b.

GODOY, A. A pesquisa qualitativa e sua utilização em administração de empresas. Revista de Administração de Empresas, v.35, n.4, p.65-71, 1995.

HARRISON, R. L.; REILLY, T. M. Mixed methods designs in marketing research. Qualitative Market Research: An International Journal, v.14, n.1, p.7-26, 2011.

HESSE-BIBER, S. Qualitative approaches to mixed methods practice. Qualitative Inquiry, v.16, n.6, p.455-468, 2010.

HUNT, S. D. The nature and scope of marketing. Journal of Marketing, v.40, n.3, p.17-28, 1976.

HUNT, S. D. The influence of philosophy, philosophies, and philosophers on a marketer's scholarship. Journal of Marketing, v.64, n.4, p.117-124, 2001.

HUNT, S. D. Foundations of marketing theory: Toward a general theory of marketing. Armonk: M.E Sharpe, 2002.

HUNT, S. D. Controversy in marketing theory: for reason, realism, truth and objectivity. Armonk: M.E Sharpe, 2003.

HUY, Q. N. Improving the odds of publishing inductive qualitative research in premier academic journals. The Journal of Applied Behavioral Science, v.48, n.2, p.282-287, 2012.

IPHOFEN, R. Ethical decision making in qualitative research. Qualitative Research, v.11, n.4, p.443-446, 2011.

JAPIASSU, H. O mito da neutralidade científica. Rio de Janeiro: Imago, 1975.

JAWORSKI, B. J. On managerial relevance. Journal of Marketing, v.75, n.1, p.211-224, 2011. 
JONASSEN, C. T. Contributions of sociology to marketing. Journal of Marketing, v.24, n.4, p.29-35, 1959.

KELEMEN, M.; BANSAL, P. The conventions of management research and their relevance for management practice. British Journal of Management, v.13, n.2), p.97-108, 2002.

KORO-LJUNGBERG, M. Validity, responsibility, and aporia. Qualitative Inquiry, v.16, n.8, p.603-610, 2010.

KORO-LJUNGBERG, M. Researchers of the world, create! Qualitative Inquiry, v.18, n.9, p.808-818, 2012.

KOVACS, M. H.; LEÃO, A. L. M.; VIEIRA, R. S. G.; BARBOSA, L.; DIAS, C. M. Podemos confiar nos resultados de nossas pesquisas? Uma avaliação dos procedimentos metodológicos nos artigos de marketing do EnANPAD. Anais do Encontro de Marketing da ANPAD, Porto Alegre, RS, Brasil, 2004.

LARA, T. A. Caminhos da razão no ocidente: A filosofia ocidental, do Renascimento aos nossos dias. (2 ${ }^{\mathrm{a}}$ ed). Petrópolis: Vozes, 1986.

LAZER, W.; KELLEY, E. J. (1960). Interdisciplinary horizons in marketing. Journal of Marketing, 25(4), 24-30.

LEGRECO, M.; TRACY, S. J. Discourse tracing as qualitative practice. Qualitative Inquiry, v.15, n.9, p.1516-1543, 2009.

LEVY, S. J. History of qualitative research methods in marketing. In Belk, R. W. (Ed.). Handbook of qualitative research methods in Marketing (p. 3-16). Northampton: Edwar Elgar, 2006.

LIMA, J. B. Pesquisa qualitativa e qualidade na produção científica em administração de empresas. Anais do Encontro Nacional da Associação Nacional de Pós-Graduação e Pesquisa em Administração. Foz do Iguaçu, PR, Brasil, 1999.
MATTOS, P. L. C. L. Os resultados desta pesquisa (qualitativa) não podem ser generalizados : pondo os pingos nos is de tal ressalva. Cadernos EBAPE.BR, v.9, edição especial, p.450-468, 2011.

McCRACKEN, G. The long interview. Thousand Oaks: Sage, 1988.

MacLARAN, P., SAREN, M., STERN, B.; TADAJEWSKI, M. The Sage handbook of marketing theory. London: Sage, 2010.

MAZZON, J. A.; HERNANDEZ, J. M. da C. Produção científica brasileira em marketing no período 2000-2009. Revista de Administração de Empresas, v.53, n.1, p.67-80, 2013.

MENDES, L., VIEIRA, F. G. D.; CHAVES, C. J. A. Responsabilidade social e isomorfismo organizacional: o paradoxo de ações (anti)éticas em busca de legitimidade no mercado brasileiro. Gestão.Org - Revista Eletrônica de Gestão Organizacional, v.7, n.2, p.192212, 2009.

MILES, M. B.; HUBERMAN, A. M. Qualitative data analysis: An expanded sourcebook. ( $2^{\text {nd }}$ ed). Thousand Oaks: Sage, 1994.

MOISANDER, J.; VALTONEN, A. Qualitative marketing research: A cultural approach. London: Sage, 2006.

NOVEMBER, P. Seven reasons why marketing practitioners should ignore marketing academic research. Australasian Marketing Journal, v.12, n.2, p.39-50, 2004.

OLIVA, A. (org.). Epistemologia: a cientificidade em questão. Campinas: Papirus, 1980.

O'TOOLE, P.; WERE, P. Observing places: using space and material culture in qualitative research. Qualitative Research, v.8, n.5, p.616-634, 2008.

PAIVA JR., F. G., LEÃO, A. L. M. DE S.; MELLO, S. C. B. de. Validade e 
confiabilidade na pesquisa qualitativa em administração. Revista de Ciências da Administração, v.13, n.31, p.190-209, 2011.

PAULUS, T. M., WOODSIDE, M.; ZIEGLER, M. F. "I tell you, it's a journey, isn't it?" Understanding collaborative meaning making in qualitative research. Qualitative Inquiry, v.16, n.10, p.852$862,2010$.

QU, S. Q.; DUMAY, J. The qualitative research interview. Qualitative Research in Accounting and Management, v.8, n.3, p.238-264, 2011.

RICHERS, R. Marketing: uma visão brasileira. São Paulo: RReCA Editora, 2000.

ROCHA, A.; ROCHA, E. Paradigma interpretativo nos estudos de consumo: retrospectiva, reflexões e uma agenda de pesquisas para o Brasil. Revista de Administração de Empresas, v.47, n.1, p.71-80, 2007.

ROSSI, C. A. V.; FARIAS, S. A. Conhecimento científico em marketing no Brasil: perspectivas para o desenvolvimento da pesquisa e teoria. Revista de Administração de Empresas, 46(4), 10-12, 2006.

ROULSTON, K. Considering quality in qualitative interviewing. Qualitative Research, v.10, n.2, p.199-228, 2010.

SANDERS, C. B.; CUNEO, C. J. Social reliability in qualitative team research. Sociology, v.44, n.2, p.325-343, 2010.

SAMPAIO, C. H.; PERIN, M. G.; LUCE, F. B.; SANTOS, M. J. dos; SANTINI, F. O. de; OLIVEIRA, M. O. R. de; LENZ, G. de S. Pesquisa científica da área de marketing no Brasil: uma revisão da primeira década do século 21 . Revista de Administração Contemporânea, v.16, n.3, p.459-478, 2012.
SAMPAIO, C. H.; PERIN, M. G. Pesquisa científica da área de marketing: uma revisão histórica. Revista de Administração Contemporânea, v.10, n.2, p.179-202, 2006.

SAUERBRONN, F. F.; FARIA, A. A utilização do método histórico em pesquisa acadêmica de marketing. Estratégia e Negócios, v.2, n.2, p.77-95, 2009.

SAUERBRONN, J. F. R.; AYROSA, E. A. T. Sobre convergência e a prática metodológica do interacionismo interpretativo na pesquisa acadêmica de marketing. Revista de Administração Contemporânea, v.14, n.5, p.854-870, 2010 .

SAUERBRONN, J. F. R.; CERCHIARO, I. B.; AYROSA, E. A. T. Uma discussão sobre métodos alternativos em pesquisa acadêmica em marketing. Gestão e Sociedade, v.5, n.12, p.254-269, 2012.

SAUNDERS, J.; LEE, N. Whiter research in marketing? European Journal of Marketing, v.39, n.3-4, p.245-260, 2005.

SHETH, J. N.; GARDNER, D. M.; GARRETT, D. E. Marketing theory: evolution and evaluation. New York: Wiley, John e Sons, 1988.

SHETH, J. N. Impact of emerging markets on marketing: rethinking existing perspectives and practices. Journal of Marketing, v.75, n.1, p.166-182, 2011.

SOUZA NETO, A. F.; MELLO, S. C. B. Olhando além do "primeiro estruturalismo" para a pesquisa em marketing. Revista de Administração Contemporânea, v.13, n.4, p.525-544, 2009.

STALLER, K. M. Qualitative research encyclopedia of research design. London: Sage, 2010.

TAYLOR, F. W. Princípios de administração científica. São Paulo: Atlas, 1995. 
TILLEY, L.; WOODTHORPE, K. Is it the end for anonymity as we know it? A critical examination of the ethical principle of anonymity in the context of 21 st century demands on the qualitative researcher. Qualitative Research, v.11, n.2, p.197212, 2011.

TRACY, S. J. Qualitative quality: eight "big-tent" criteria for excellent qualitative research. Qualitative Inquiry, v.16, n.10, p.837-851, 2010.

VARADARAJAN, P. R. Musings on relevance and rigor of scholarly research in marketing. Journal of the Academy of Marketing Science, v.31, n.4, p.368-376, 2003.

VIEIRA, F. G. D. Por quem os sinos dobram? Uma análise da publicação científica na área de marketing do ENANPAD. Anais do Encontro Nacional da Associação Nacional de Pós-Graduação e Pesquisa em Administração, Florianópolis, SC, Brasil, 1998.

VIEIRA, F. G. D. O fim da academia: por que é difícil produzir conhecimento em administração no Brasil? Anais da Semana do Administrador, Maringá, PR, Brasil, 2000a.

VIEIRA, F. G. D. Panorama acadêmicocientífico e temáticas de estudos de marketing no Brasil. Anais do Encontro Nacional da Associação Nacional de PósGraduação e Pesquisa em Administração, Florianópolis, SC, Brasil, 2000b.

VIEIRA, F. G. D.; Crubellate, J. M.; Silva, I. G.; Silva, W. R. Silêncio e omissão: aspectos da cultura brasileira nas organizações. RAE Eletrônica (Online), v.1, n.1, p.1-14, 2002.

VIEIRA, F. G. D. Narciso sem espelho: a publicação brasileira de marketing. Revista de Administração de Empresas, v.43, n.1, p.81-90, 2003.
VIEIRA, F. G. D. Latindo atrás do Lattes. Revista Eletrônica Espaço Acadêmico, v.7, n.73, p.1-4, 2007.

VIEIRA, F. G. D. Sabemos a lição, mas ainda não aprendemos: comentários sobre a pesquisa científica da área de marketing, no Brasil. Revista de Administração Contemporânea, v.16, n.3, p.484-488, 2012.

VIEIRA, V. A; TIBOLA, F. Pesquisa qualitativa em marketing e suas variações: trilhas para pesquisas futuras. Revista de Administração Contemporânea, v.9, n.2, p.9-33, 2005.

WATSON, C. The 'impossible vanity': uses and abuses of empathy in qualitative inquiry. Qualitative Research, v.9, n.1, p.105-117, 2009.

WENSLEY, R. A bridge over troubled water? European Journal of Marketing, v.36, n.3, p.391-400, 2002.

WILES, R.; CROW, G.; PAIN, H. Innovation in qualitative research methods: a narrative review. Qualitative Research, v.11, n.5, p.587-604, 2011.

WINICK, C. Anthropology's contributions to marketing. Journal of Marketing, v.26, n.3, p.53-60, 1961.

YIN, R. K. Estudo de Caso planejamento e método. Porto Alegre: Bookman, 2001.

ZALTMAN, G. Theory construction in marketing: some thoughts on thinking. New York: John Wiley, 1982. 